\title{
Erratum to: Perfusion changes in gastric adenocarcinoma: evaluation with 64-section MDCT
}

Jin Yao, ${ }^{1}$ Zhi-gang Yang, ${ }^{1,2}$ Tian-wu Chen, ${ }^{1}$ Yuan Li, ${ }^{1}$ Lin Yang ${ }^{1}$

${ }^{1}$ Department of Radiology, West China Hospital, Sichuan University, 37\# Guo Xue Xiang, Chengdu, Sichuan 610041, China ${ }^{2}$ National Key Laboratory of Biotherapy Center, West China Hospital, Sichuan University, 37\# Guo Xue Xiang, Chengdu, Sichuan 610041, China

\section{Erratum to: Abdom Imaging (2010) 35:195-202 DOI 10.1007/s00261-009-9503-1}

In the Tables $1,2,3$, and 5 , the unit of perfusion was stated incorrectly as $\mathrm{mL} / 100 \mathrm{~g} / \mathrm{min}$. It should have been stated as $\mathrm{mL} / \mathrm{min} / \mathrm{mL}$. The corrected tables are as follows:

Table 1. Intraobserver variability for PF, PE, TTP, and BV measurement of normal stomach

\begin{tabular}{llrrr}
\hline Parameter measurement & ICC $^{\mathrm{a}}(95 \% \mathrm{CI})$ & Mean difference & SD & $95 \%$ limits of agreement \\
\hline PF $(\mathrm{mL} / \mathrm{min} / \mathrm{mL})$ & $0.929(0.894-0.952)$ & -1.44 & 13.69 & $-28.27,25.38$ \\
PE (Hu) & $0.969(0.954-0.979)$ & -0.29 & 8.24 & $-16.45,15.87$ \\
TTP $(\mathrm{s})$ & $0.816(0.726-0.877)$ & 0.21 & 10.22 & $-19.83,20.25$ \\
BV $(\mathrm{mL} / 100 \mathrm{~g})$ & $0.936(0.905-0.957)$ & 0.24 & 5.81 & $-11.14,11.62$ \\
\hline
\end{tabular}

${ }^{\mathrm{a}} \mathrm{ICC}$, intraclass correlation coefficient

Table 2. Intraobserver variability for PF, PE, TTP, and BV measurement of gastric adenocarcinoma

\begin{tabular}{llccc}
\hline Parameter measurement & ICC $(95 \% \mathrm{CI})$ & Mean difference & SD & $95 \%$ limits of agreement \\
\hline PF $(\mathrm{mL} / \mathrm{min} / \mathrm{mL})$ & $0.922(0.868-0.954)$ & 2.38 & 8.05 & $-13.39,18.15$ \\
PE (Hu) & $0.990(0.984-0.994)$ & 0.10 & 2.25 & $-4.30,4.51$ \\
TTP $(\mathrm{s})$ & $0.969(0.948-0.982)$ & -1.21 & 4.20 & $-9.45,7.03$ \\
BV $(\mathrm{mL} / 100 \mathrm{~g})$ & $0.968(0.947-0.981)$ & -0.68 & 5.23 & $-10.94,9.58$ \\
\hline
\end{tabular}

${ }^{\mathrm{a}} \mathrm{ICC}$, intraclass correlation coefficient

The online version of the original article can be found under doi:10.1007/s00261-009-9503-1.

Correspondence to: Zhi-gang Yang; email: yangzg1117@yahoo.com.cn 
Table 3. Perfusion values in gastric adenocarcinoma vs. in normal stomach

\begin{tabular}{|c|c|c|c|c|}
\hline Location & Perfusion parameter & Tumor $^{\mathrm{a}}$ & Normal stomach ${ }^{\mathrm{a}}$ & $P$ value \\
\hline \multirow[t]{4}{*}{ Cardia } & $\mathrm{PF}(\mathrm{mL} / \mathrm{min} / \mathrm{mL})$ & $28.87 \pm 11.50$ & $24.58 \pm 20.91$ & 0.45 \\
\hline & $\mathrm{PE}(\mathrm{Hu})$ & $24.51 \pm 8.94$ & $20.55 \pm 11.49$ & 0.20 \\
\hline & TTP (s) & $29.98 \pm 13.12$ & $31.42 \pm 15.22$ & 0.55 \\
\hline & $\mathrm{BV}(\mathrm{mL} / 100 \mathrm{~g})$ & $19.78 \pm 21.02$ & $12.35 \pm 12.93$ & 0.14 \\
\hline \multirow[t]{4}{*}{ Body } & $\mathrm{PF}(\mathrm{mL} / \mathrm{min} / \mathrm{mL})$ & $31.73 \pm 19.56$ & $40.98 \pm 30.29$ & 0.19 \\
\hline & $\mathrm{PE}(\mathrm{Hu})$ & $29.49 \pm 11.70$ & $35.28 \pm 29.95$ & 0.48 \\
\hline & TTP (s) & $31.28 \pm 11.54$ & $30.18 \pm 8.89$ & 0.75 \\
\hline & BV (mL/100 g) & $16.02 \pm 7.02$ & $15.47 \pm 11.13$ & 0.83 \\
\hline \multirow[t]{4}{*}{ Antrum and pylorus } & $\mathrm{PF}(\mathrm{mL} / \mathrm{min} / \mathrm{mL})$ & $28.02 \pm 14.96$ & $30.35 \pm 14.40$ & 0.59 \\
\hline & $\mathrm{PE}(\mathrm{Hu})$ & $28.67 \pm 15.87$ & $29.02 \pm 12.23$ & 0.93 \\
\hline & TTP (s) & $34.77 \pm 12.23$ & $34.29 \pm 11.71$ & 0.89 \\
\hline & $\mathrm{BV}(\mathrm{mL} / 100 \mathrm{~g})$ & $20.80 \pm 12.41$ & $11.29 \pm 9.99$ & 0.006 \\
\hline \multirow[t]{4}{*}{ Whole stomach } & $\mathrm{PF}(\mathrm{mL} / \mathrm{min} / \mathrm{mL})$ & $30.28 \pm 14.89$ & $32.85 \pm 25.69$ & 0.49 \\
\hline & $\mathrm{PE}(\mathrm{Hu})$ & $27.89 \pm 11.29$ & $29.06 \pm 23.30$ & 0.72 \\
\hline & TTP (s) & $32.60 \pm 12.30$ & $31.46 \pm 11.86$ & 0.57 \\
\hline & $\mathrm{BV}(\mathrm{mL} / 100 \mathrm{~g})$ & $19.75 \pm 14.74$ & $13.59 \pm 11.46$ & 0.004 \\
\hline
\end{tabular}

${ }^{\mathrm{a}}$ Data are means \pm standard deviations

Table 5. Perfusion values of subgroups with lymph node metastases vs. without lymph node metastases

\begin{tabular}{llll}
\hline Perfusion parameter & With lymph node metastases $(n=29)^{\mathrm{a}}$ & Without lymph node metastases $(n=13)^{\mathrm{a}}$ & $P$ value \\
\hline PF $(\mathrm{mL} / \mathrm{min} / \mathrm{mL})$ & $30.67 \pm 12.36$ & $31.10 \pm 21.35$ & 0.93 \\
PE $(\mathrm{Hu})$ & $29.32 \pm 9.90$ & $25.44 \pm 11.17$ & 0.29 \\
TTP $(\mathrm{s})$ & $32.58 \pm 11.99$ & $32.55 \pm 16.51$ & 0.99 \\
BV $(\mathrm{mL} / 100 \mathrm{~g})$ & $18.75 \pm 9.89$ & $15.62 \pm 11.15$ & 0.39 \\
\hline
\end{tabular}

${ }^{\mathrm{a}}$ Data are means \pm standard deviations 